\title{
Estado del componente bucal de la salud en la República Argentina. Informe pre pandemia
}

\section{Status of the oral component of health in the Argentine Republic. Pre pandemic report}

\begin{abstract}
Resumen
El propósito del presente artículo es analizar parte de la situación de salud bucal en Argentina. Un estado federal descentralizado, conformado por veinticuatro jurisdicciones con autonomía política, veintitrés provincias y una ciudad autónoma. La caries dental afecta a más de la mitad de la población y es la patología más frecuente en los niños en edad escolar. El CPOD promedio a los 12 años es de 5,11. Cada año se confirman 3000 nuevos casos de cáncer bucal. La Dirección Nacional de Salud Bucodental se reconoce como rectora de las acciones referentes al componente bucal de la salud e implementa los siguientes programas: Programa Nacional de Cáncer Bucal, Programa Nacional de Rehabilitación Bucodental y Programa de Prevención y Asistencia Primaria Bucodental. Centrados en fortalecer el primer nivel de atención con un modelo de capacidad resolutiva con equipos transdisciplinarios, desarrollar un sistema de monitoreo y unificar el registro clínico odontológico con consentimiento del paciente.
\end{abstract}

Palabras clave: Salud pública; Población vulnerable; Salud bucal; Argentina (fuente: DeCS BIREME).

\begin{abstract}
The purpose of this article is to analyze part of the oral health situation in Argentina. A decentralized federal state, made up of twenty-four jurisdictions with political autonomy, twenty-three provinces, and an autonomous city. Dental caries affects more than half of the population and is the most common pathology in school-age children. The average DMFT at 12 years is 5.11. Every year 3000 new cases of oral cancer are confirmed. The National Directorate of Bucodental Health is recognized as the rector of the actions related to the oral health component and implements the following programs: National Oral Cancer Program, National Oral Rehabilitation Program and Prevention and Primary Oral Assistance Program. Focused on strengthening the first level of care with a model
\end{abstract}

(C) Los autores. Este artículo es publicado por la revista Odontología Sanmarquina de la Facultad de Odontología, Universidad Nacional Mayor de San Marcos. Este es un artículo de acceso abierto, distribuido bajo los términos de la licencia Creative Commons Atribucion - No Comercia_Compartir Igual 4.0 Internacional. (http://creativecommons.org/licenses/by-nc-sa/4.0/) que permite el uso no comercial, distribución y reproducción en cualquier medio, siempre que la obra original sea debidamente citada. 
of resolute capacity with transdisciplinary teams, developing a monitoring system and unifying the dental clinical record with the consent of the patient.

Keywords: Public health; Vulnerable population; Oral health; Argentina (source: MeSH NLM).

\section{Introducción}

Las disparidades en Salud son las diferencias de salud en la población específica (en la prevalencia de la enfermedad, los resultados de salud, o el acceso al cuidado de salud) que son evitables e injustas cuando son consideradas a partir de las perspectivas de justicia social y de los derechos humanos ${ }^{1}$.

Aunque ha habido mejoras importantes en la salud bucal durante las últimas décadas, por las investigaciones que generaron notables avances sobre la prevención y tratamiento de enfermedades, las desigualdades en salud bucal siguen siendo, en el marco de lo social, similares a la de la salud general ${ }^{2}$.

El componente bucal de la salud es un indicador socioeconómico y de la conducta que debe ser analizado al estudiar las inequidades en salud ${ }^{3}$.

A nivel mundial, la mayor carga de enfermedad bucal se encuentra en las poblaciones pobres ${ }^{4}$.

La caries dental, es un problema grave de salud pública. Su impacto en las personas y comunidades es considerado en términos de dolor y sufrimiento, deterioro de la función, y reducción de la calidad de la vida. A nivel mundial, la mayor prevalencia de las enfermedades orales radica en las poblaciones desfavorecidas y pobres. El patrón actual de la enfermedad bucal refleja distintos perfiles de riesgo entre los países, relacionados con las condiciones de vida, factores conductuales y ambientales, los sistemas de salud y la implementación de planes para prevenir la enfermedad bucal ${ }^{5-6}$. Es la enfermedad crónica más común en infante, cinco veces más común que el asma. siete veces más que la fiebre y 14 veces más que la bronquitis crónica ${ }^{7}$.

Los reportes epidemiológicos muestran que la prevalencia de caries de la temprana infancia es de aproximadamente $70 \%$ en países en vías de desarrollo y del 1 al 12 $\%$ en desarrollados ${ }^{7}$.

Según el centro de Control de Enfermedades (CDC) la caries dental es una disbiosis controlable y posible de prevenir, pero sigue siendo la enfermedad crónica más común en la niñez. En países económicamente desarrollados se produce una polarización, de tal forma que el impacto de la enfermedad es padecido por las personas con menores recursos económicos, menos educación, de minorías raciales y étnicas que constituyen la franja más desposeída ${ }^{8}$.

La caries dental es una enfermedad crónica común que resulta de desequilibrio de múltiples factores de riesgo, tanto biológicos como conductuales a través del tiempo. Se define caries de la primera infancia (ECC) a la presencia de uno o más lesiones de caries en superficies diente primario en un niño de 71 meses de edad o menor ${ }^{9}$.

Diferentes autores demostraron que las lesiones iniciales de caries comienzan con la aparición de los primeros dientes en el medio cariogénico y aumenta en prevalencia y gravedad hasta los 6 años, experimentando dolor bucal. A pesar de un mayor conocimiento y recursos de salud entre las madres de zonas urbanas, el mayor acceso de los niños de poblaciones urbanas a la comida azucarada y la frecuencia de consumo se asociaron con una mayor prevalencia y gravedad de las caries comparadas con los niños en zonas rurales. La caries severa se asocia en la literatura con la desnutrición. Se necesitan estrategias de promoción y prevención en la primera infancia para incorporar la educación nutricional y de salud bucal y el cuidado dental en los servicios de salud maternoinfantil, y desarrollar políticas para prohibir la venta de comida cariogénica en las escuelas ${ }^{7-12}$.

Las enfermedades gingivoperiodontales son un problema de salud pública por su alta prevalencia, los daños que ocasiona, el tratamiento costoso y por ser susceptibles de prevenirse. A pesar de no existir revisiones sistemáticas de estudios de prevalencia de periodontitis, según lo publicado, si podemos establecer que los niños presentan gingivitis que aumenta con la edad, y que la población en general presente un alto componente inflamatorio en las encías ${ }^{13}$.

Las enfermedades periodontales son la respuesta inflamatoria de los tejidos ante los productos del biofilm dental, que depende de las características de factores locales y sistémicos del hospedero, además del periodo de tiempo en que esté presente el biofilm de placa bacteriana. En niños y adolescentes su manifestación más común es la gingivitis, inflamación no específica de la encía marginal ${ }^{14}$.

En la población adolescente la evidencia muestra la destrucción de tejidos periodontales.

En los adultos, son responsable en gran medida de la pérdida de dientes. Los indicadores de riesgo evaluables son: el grupo etario, el género, la escolaridad, el nivel socioeconómico, el sistema de salud y su posibilidad de acceso, el tabaquismo y la relación existente con enfermedades crónicas no transmisibles ${ }^{14}$.

Según la Organización Mundial de la Salud una de las enfermedades no transmisibles de incumbencia en la salud bucodental y general es el cáncer. El cáncer bucal es una enfermedad altamente invasiva, que afecta a personas de todas las edades, representando un 5\% del total de los cánceres diagnosticados a nivel mundial. 
El cáncer presenta una importante morbilidad a nivel mundial. Se estima que el número de casos nuevos pasará de 10 millones registrados en el año 2000 a 15 millones antes del 2020. El 60\% de estos se presentarán en los países menos desarrollados. Actualmente los casos nuevos, registrados fueron 4,7 millones en los países desarrollados y casi 5,5 millones en los menos desarrollados ${ }^{15}$.

Los servicios de salud ofrecen asistencia curativa basada en la complejidad de las nuevas tecnologías para realizar intervenciones. Los organismos internacionales han repetido los llamamientos a los ministerios de sanidad de cada uno de los países, solicitando un cambio en el perfil de sus servicios hacia la Salud y no solo hacia el tratamiento de las secuelas de la enfermedad con sus prestaciones.

Según la OPS casi todos los países de la región, han alcanzado una buena cobertura de intervenciones materno infantiles. No obstante, la situación socioeconómica y los otros determinantes sociales generan desigualdades, que tienen una fuerte incidencia sobre la salud y el acceso a los servicios ${ }^{16-17}$.

Según Sheiham y cols. el componente bucal de la salud es parte integral de la salud, las enfermedades que afectan la cavidad oral tienen repercusión no solo en la salud física sino también en la mental de los individuos. Numerosos estudios demostraron la asociación entre las afecciones orales y un peso corporal bajo, trastornos del sueño y de la alimentación debidos al dolor y cambios en los procesos digestivos ${ }^{18}$.

\section{República Argentina}

Argentina presenta un mapa heterogéneo con diversas realidades. Es un estado federal descentralizado, integrado por el Estado Nacional conformado por veinticuatro jurisdicciones con autonomía política, veintitrés provincias y una ciudad autónoma. Geográficamente se divide en cinco regiones: Noroeste, Noreste, Cuyo, Centro y Sur. La forma de gobierno es representativa, republicana y federal. De esta manera se presentan cinco Regiones Sanitarias.

De acuerdo al Censo Nacional de Población, Viviendas y Hogares del año 2010 en la República Argentina había 40117096 habitantes. Según las proyecciones del Instituto de Censos Nacional al 2019 la población asciende a 44938712 habitantes. Es un país con baja densidad de población, muy concentrada en el conurbano de Gran Buenos Aires $(38,9 \%)^{19-21}$.

Existe consenso en considerar a la pobreza como heterogénea, es decir, no es igual en todas las regiones y las personas que la sufren la viven de manera distinta, dependiendo del contexto del cual forman parte. También es multidimensional, ya que involucra al ser humano en todas sus dimensiones: sociales, psicológicas, culturales, ideológicas, espirituales, intelectuales. Los grupos de hogares con privación se distinguen según el tipo y la intensidad de sus carencias, por lo que existe variedad en cuanto métodos para medirla.
Argentina registró una tasa de mortalidad infantil de $9,6 \%$ en el año 2013. La tasa de pobreza en 2018 , se ubicaba en 33,6\%. La desocupación es del 10,1\% (primer trimestre 2019) ${ }^{22}$.

El Observatorio de la Deuda Social de la Universidad Católica Argentina (UCA) analizando el tercer trimestre de 2019, registró que el 59,5\% de los niños/as y adolescentes viven en hogares con ingresos por debajo de la línea de pobreza ${ }^{23}$.

En 2016, la cobertura de instalaciones de agua potable de la red pública era de $84,4 \%$, mientras que el acceso a cloacas era de $58,4 \%{ }^{24}$.

Los niños y niñas de 5 a 11 años conforman el 39,9\% de quienes no tienen garantizado el derecho a agua, el $72,5 \%$ es de los niveles socioeconómicos muy bajo y bajo ${ }^{25}$.

Los sistemas de cobertura social y proyección son el mecanismo para enfrentar las desigualdades para permitir a las personas evitar la privación de acceso al sistema, considerado inaceptable en la sociedad. Compensan la ausencia de los ingresos del trabajo, proporcionar atención médica, vivienda, agua potable y saneamiento, educación o trabajo social. Puede tratarse de ayudas en efectivo o en coberturas, que van de pensiones a eximiciones de gastos de usuario a servicios. En la Argentina, en los fundamentos del estado de bienestar, las personas recorrían la ruta del empleo obteniendo la protección necesaria frente a distintas situaciones, como la enfermedad o el retiro a través de la Obra Social. Ser beneficiario de una obra social es un indicador indirecto de empleo formal.

El sector salud argentino está integrado por tres subsistemas: a) público; b) seguridad social (obras sociales), y c) privado. La seguridad social también incluye fondos y seguros sociales nacionales y provinciales junto con el Instituto Nacional de Servicios Sociales para Jubilados y Pensionados (INSSJyP) comúnmente conocido como PAMI; Se describe como un sistema fragmentado, heterogéneo y desigual, tanto por su organización y financiamiento como por el acceso que brinda a los servicios de salud ${ }^{23}$.

Para el año 2010, según el último censo poblacional, el $64 \%$ de los habitantes contaba con una cobertura, siendo la seguridad social la mayoritaria (46\%); el $16 \%$ contaba con una prepaga (privado), esta última ya sea a través de la obra social o por contratación voluntaria; el $2 \%$ estaba registrado en programas y planes estatales de salud y el $36 \%$ no contaba con ninguna cobertura de salud y dependiendo exclusivamente de los servicios públicos sanitarios (Indec 2010) ${ }^{20}$.

Existen seguros voluntarios de empresas de salud con sistema prepago, que son instituciones del sector privado, en su mayoría con fines de lucro ${ }^{24}$.

Los hospitales públicos, los centros de atención primaria de salud (CAPS) y postas sanitarias representan la salud pública. 
Dada la estructura federal del país los gobiernos provinciales se hacen cargo del financiamiento y de la provisión de servicios del sector público. El registro de los datos de financiamiento con establecimientos, la mayor parte de los centros de salud depende de las provincias (59\%), seguidos por los municipios (39\%) y, por último, la Nación $(2 \%)^{24}$.

La Argentina no cuenta con sistemas de vigilancia consolidados. No obstante, pueden presentarse algunos comentarios referidos a ciertos aspectos que ayuden a determinar las diferencias epidemiológicas y regionales.

\section{Componente bucal de la salud}

En Argentina existen 18 universidades de carácter público y privado que ofrecen la carrera de Odontología donde egresan profesionales con sólida formación científico técnica.

En Argentina los datos sobre prevalencia son pocos, pero no difieren de los demás países de la región, Piovano y colaboradores ${ }^{27}$, sobre una población de 848 niños de 2 a 5 años de edad, encontraron a la edad de 2 años 3,5 superficies con caries y a los 5 años 10 superficies. En este grupo etario solo el 22,4\% estaba libre de caries. A los 5 años el componente cd del ceod, indicador de presencia de caries en dentición primaria considerando la unidad diente, fue de $5,46 \pm 0,21 \mathrm{y}$ el cs, indicador de caries según superficie dentaria con caries, alcanzó el $10,04 \pm 0,5$.

El reporte anual 2014 del Programa Nacional de Salud Escolar (PROSANE) muestra que "los problemas de salud detectados en los/as niños/as y adolescentes se presentaron con distinta frecuencia. Los más prevalentes fueron los hallazgos odontológicos. Más de la mitad de los/as niños/as evaluados /as presentaron patología de las piezas dentarias (caries, dientes obturados, extraídos y perdidos), tanto de las piezas dentarias temporales como permanentes. Este problema afecta a más de la mitad de la población y es la patología más frecuente en los/as niños/as en edad escolar. La maloclusión fue muy frecuente y alcanzó el 18\%". Seguido del sobrepeso y la obesidad y la disminución de la agudeza visual. Según la región se registró entre un 45 y $63 \%$ de la población afectada de patología dental ${ }^{28}$.

El CPOD promedio a los 12 ańos es de 5.11 registrado en una población de 7698 niños ${ }^{29}$.

La Organización Mundial de la Salud en el año 1978, entre las metas para el año 2000, estableció como deseable alcanzar una media de CPOD de 3 o menos a la edad de 12 años ${ }^{30}$. En el año 2006, el Comité Ejecutivo de la Organización Panamericana de la Salud planteó las siguientes categorías para establecer perfiles de caries en esa franja etaria: país emergente $(\mathrm{CPOD}>5)$, en crecimiento (CPOD 3-5) y en consolidación (CPOD < 3 ), todos con algún tipo de programa de fluorización ${ }^{31}$. Desde esta perspectiva, los datos encontrados permiten encuadrar el perfil de caries de la muestra global estudiada por la Dirección Nacional de Salud Bucodental como perteneciente al grupo país emergente.
El Observatorio de la Deuda Social Argentina expresa que en el año 2015 el 48\% de los niños carecían de cobertura de salud, y en el mismo informe mostraba que el $47 \%$ presentaban déficit de control odontológico. Esta situación deja expuesto un problema social relacionado con la educación sobre hábitos de nutrición e higiene y reconocimiento del componente bucal de la salud. El deterioro o la pérdida de piezas dentarias no es percibida por las familias como enfermedad, sino como un proceso lógico y natural ${ }^{22}$.

La Dirección Nacional de Salud Bucodental en su informe anual, manifiesta que en nuestro país se confirman 3000 nuevos casos de cáncer bucal al año ${ }^{29}$.

La Organización Panamericana de la Salud (OPS 2017) recomienda para mejorar aún más la salud bucodental en la Región de las Américas : a) reconocer la salud bucodental como un factor que repercute directamente en la calidad de vida de la población que está envejeciendo; b) fortalecer la capacidad de los trabajadores de atención primaria de salud; c) abordar el estado de la ciencia con respecto al cáncer de la cavidad bucal asociado al virus de papiloma humano (VPH) y sus alcances en los proyectos de investigación y las políticas de salud pública que se aborden en un futuro en la región ${ }^{17}$.

El ministerio de Salud de la Nación cuenta con una Dirección Nacional de Salud Bucal creada bajo el Decreto $580 / 2015^{29}$

La Dirección Nacional de Salud Bucodental (DINA$\mathrm{SAB}$ ) se reconoce como rectora de las acciones referentes al componente bucal de la salud para brindar unificación en cuanto a evaluación diagnóstica (registro) y protocolos de atención en los diferentes programas, tanto del Ministerio de Salud de la Nación como los Ministerios Provinciales y direcciones locales, para aunar esfuerzos economizando recursos que permitan el acceso a la salud de la población.

\section{Programas nacionales}

La Dirección Nacional de Salud Bucodental (DINASAB) implementa nacionalmente los siguientes programas ${ }^{29}$ :

Programa Nacional de Cáncer Bucal. Su objetivo principal es:

- Disminuir la morbimortalidad por cáncer bucal en todo el territorio argentino, generar una red asistencial de contención, promover estilos de vida saludables en la población y conformar un registro nacional de lesiones precancerosas y cáncer oral, estableciendo relaciones etiológicas y epidemiológicas de las distintas regiones del país (investigación).

- Trabajar en los primeros dos niveles de prevención en esta enfermedad, garantiza mejor pronóstico y calidad de vida para los pacientes, y menores costos para el sistema público de salud.

Programa Nacional de Rehabilitación Bucodental. Su objetivo principal es: desarrollar un sistema de atención 
odontológica de rehabilitación bucodental que promueva la inclusión social y logre el acceso universal de poblaciones de pacientes desdentados a la asistencia oportuna e integral. Para ello se trabaja articuladamente con los programas de Prevención y Asistencia Primaria Bucodental y Cáncer Bucal.

Programa de Prevención y Asistencia Primaria Bucodental. El objetivo general del Programa es contribuir a la igualdad de oportunidades previniendo y resolviendo problemas de salud, de modo de mejorar el rendimiento e inserción de la población en desventaja social, económica, psicológica y biológica.

Sus tres pilares son:

\section{Programa Nacional de Salud Bucodental en la Prime-} ra Infancia. Este incluye acciones tendientes a promover:

- Interacción con los programas de maternidad e infancia del ministerio, desde la madre embarazada cubriendo los primeros mil días.

- Atención odontológica integral a preescolares (2 a 5 años de edad), comprende acciones educativas, preventivas y curativas logrando el alta básica (control de la infección) para permitir su ingreso a la escolaridad primaria con bajo nivel de infección bucal. Se desarrollará la atención en los jardines estatales, los profesionales regionales son entrenados para implementar la técnica de restauración atraumática (TRA) patrocinada por la OMS para la atención de poblaciones vulnerables, se realiza la aplicación de fluoruros tópicos (barnices y geles según el nivel de riesgo cariogénico).

Programa Nacional de Salud Bucodental en Escolares. Este incluye acciones tendientes a promover:

- Evaluación de estado de salud bucal a escolares (6 a 13 años), derivación a los centros de atención primaria de salud de los municipios a los niños que presenten patologías para lograr el alta básica (incluye promoción de salud, control de cepillado, inactivación de caries, aplicación de fluoruros). Implementación de un programa de promoción de salud en conjunto con el ministerio de educación entrenando a los maestros en el cepillado diario con pastas fluoradas. Promover conductas saludables, propiciar la implementación del kiosco saludable en el establecimiento.

Programa Nacional de Salud Bucodental Atención Primaria en adolescentes y Adultos. Este incluye acciones tendientes a promover:

- Atención odontológica integral con componente educativo preventivo en los centros de atención primaria de salud (CAP) para población adolescente y adultos, implementando la resolución por altas. El centro de salud derivará los pacientes desdentados parciales o totales para poder lograr el alta integral al programa de
Rehabilitación Bucodental. donde se realizará las prótesis comunitarias.

\section{Conclusiones}

Resulta necesario fortalecer el primer nivel de atención para alcanzar la salud universal. Respetando los principios de equidad, universalidad, solidaridad, calidad, eficiencia, eficacia, bioética, con enfoque de género y generacional. Un modelo con capacidad resolutiva, con equipos de salud transdisciplinarios, y con la participación activa de referentes de otros sectores.

Desarrollar e implementar un sistema de monitoreo y evaluación de las políticas en el componente bucal de la salud.

Unificación del registro clínico odontológico, con consentimiento del paciente, conforme a la ley nacional de derechos del paciente $\mathrm{N}^{\circ}$ 26.812, implementado por aceptación de los profesionales. Capacitación para ajustar los registros a lo establecido por la ley.

\section{Referencias bibliográficas}

1. Lee JY, Divaris K. The ethical imperative of addressing oral health disparities: a unifying framework. J Dent Res. 2014;93(3):224-30.

2. Mossey P, Petersen P. Budapest Declaration. IADR-GOHIRA'J Dent Res. 2014;93(7 Suppl):120S-121S.

3. Thomson WM. Social inequality in oral health. Community Dentistry and Oral Epidemiology. 2012;40(Suppl 2):28-32.

4. Beaglehole R, Bonita R. Alcohol: a global priority. Lancet. 2009;373:2173-74.

5. Petersen PE World Health Organization global policy for improvement of oral health--World Health Assembly 2007. Int Dent J. 2008;58(3):115-21.

6. Pitts N, Amaechi B, Niederman R, Acevedo A, Vianna R, Ganss C, et al. Global Oral Health Inequalities. Dental Caries Task Group Research Agenda. Adv Dent Res. 2011;23(2):211-220.

7. American Dental Association. Statement on Early Childhood Caries. 2000. [Consultado en enero 2020]. Disponible en: http://www.ada.org/en/about-theada/ ada-positions-policies-and-statements/statement-on-early-childhood-caries.

8. Oral Health: preventing cavities, gum disease, tooth loss, and oral cancers. At a Glance 2011. Centers for Disease Control and Prevention (CDC). [Consultado diciembre 2019]. Accesible en: http://www.cdc.gov/chronicdisease/ resources/publications/AAG/doh.htm.

9. Policy on Early Childhood Caries (ECC): Classifications, Consequences, and Preventive Strategies. American Academy of Pediatric Dentistry [Internet]. 2014. [Citado el 2014]. Disponible en: http://www.aapd.org/ media/policies_guidelines/p_eccclassifications.pdf.

10. Villena Sarmiento R, Pachas Barrionuevo F, Sánchez Huaman Y, Carrasco Loyola M. Prevalencia de caries de infancia temprana en niños menores de 6 años de edad residentes en poblados urbano marginales de Lima Norte. Rev Estomatol Herediana. 2011;21(2):79-86. 
11. Sacramento SA. Californian Dental Association. [Consultado en diciembre 2019]. Accesible en: www.cda.org/ portals/0/pdfs/fact_sheets/early_childhood_caries_spanish.pdf

12. American Academy of Pediatric Dentistry. Policy on early childhood caries (ECC): classifications, consequences, and preventive strategies. Pediatr Dent. 2016;38(6):52-54.

13. Oppermann R, Haas A, Kuchembecker C, Susin C. Epidemiology of periodontal diseases in adults from Latin America. Periodontol 2000. 2015;67(1):13-33. DOI: 10.1111/prd.12061.

14. Madrigal D, Viteri EM, Romero MR, Colmenares MM, Suárez Á. Predisposing factors for gingival inflammation associated with steel crowns on temporary teeth in the pediatric population. A systematic literature review. Rev Fac Odontol Univ Antioq. 2014;26(1):152-163.

15. Organización Mundial de la Salud. 2003. [consultado en marzo 2018]. Disponible en: https://www.who.int/mediacentre/news/releases/2003/pr27/es/

16. Organización Panamericana de la Salud. Salud en las Américas+ Resumen: panorama regional y perfiles de país. [Consultado en marzo 2018]. Accesible en: https:// www.paho.org/salud-en-las-americas-2017/wp-content/ uploads/2017/09/Print-Version-Spanish.pdf

17. 160a Sesión del Comité Ejecutivo. Organización Panamericana de la Salud. Propuesta de plan regional decenal sobre salud bucodental para las Américas: Informe final. [Consultado en enero 2020]. Accesible en: https:// iris.paho.org/bitstream/handle/10665.2/34217/CE160 INF-7s.pdf? sequence $=2 \&$ isAllowed $=y$

18. Sheiham A. Oral health, general health and quality of life. Bulletin of the World Health Organization. 2005;83(9):644

19. Instituto Nacional de Estadística y Censos (INDEC). Censo Nacional de Población, Hogares y Viviendas 2001 y Censo Nacional de Población, Hogares y Viviendas 2010. [Consultado en marzo 2018]. Accesible en: https://www.indec.gob.ar/ftp/cuadros/poblacion/censo2010_tomo1.pdf

20. Instituto Nacional de Estadística y Censos (INDEC). Proyecciones provinciales de población por sexo y grupo de edad 2010- 2040. [Consultado en marzo 2018]. Accesible en: https://www.indec.gob.ar/ftp/ cuadros/publicaciones/proyeccionesyestimaciones_ nac_2010_2040.pdf

21. República de la Argentina, Ministerio de Salud, Organización Panamericana de la Salud. Indicadores básicos Argentina 2015 [Consultado en marzo 2019]. Accesible en: https://iris.paho.org/bitstream/handle/10665.2/18697/ indicadores_basicos_esp.pdf?sequence $=1$
22. Mercado de trabajo. Tasas e indicadores socioeconómicos (EPH) Tercer trimestre de 2019 ISSN 2545-6768 Instituto Nacional de Estadística y Censos (INDEC). [Consultado en diciembre 2019]. Accesible en: https://www. indec.gob.ar/uploads/informesdeprensa/mercado_trabajo_eph_3trim19BCC9AAAD16.pdf

23. Bonfiglio J, Vera J;Salvia A .La pobreza como privación más allá de los ingresos (2010-2019). Introducción de datos fundados en un enfoque de derechos - 1a ed. - Ciudad Autónoma de Buenos Aires: Educa, 2020. [Consultado en marzo 2020]. Disponible en: http:// wadmin.uca.edu.ar/public/ckeditor/Observatorio $\% 20$ Deuda\%20Social/Documentos/2020/2020-OBSERVATORIO-DOC-EST-POBREZA-PRIVACIONES-INGRESOS.pdf

24. Repetto F, Potenza dal Masseto F. Sistemas de protección social en América Latina y Caribe (LC/W.508). Buenos Aires: CEPAL; 2012.

25. PNUD (Programa de las Naciones Unidas para el Desarrollo) (2010), "Informe Nacional sobre Desarrollo Humano 2010. Desarrollo humano en Argentina: trayectos y nuevos desafíos", Buenos Aires. [consultado en enero 2020]. Accesible en: http://hdr.undp.org/sites/default/ files/argentina_indh_2010.pdf

26. Bonfiglio J, Vera J;Salvia A .Condiciones Materiales de vida. Hábitat, pobreza y desigualdad en los hogares urbanos de la Argentina (2010-2017) $1^{\circ}$ Ed. Ciudad Autonoma de Buenos Aires.Educa 2018.

27. Piovano S, Bordoni N, Doño R, Argentieri A, Cohen A, Klemonskis G, et al. Estado dentario en niños, adolescentes y adultos de la Ciudad Autónoma de Buenos Aires. Revista de la Facultad de Odontología (UBA). 2008;23(10):54-55

28. Andreacchio M, Argentatti C, Dematteis S, Finelli I, Folgueral S, González P, et al. Reporte anual 2014 análisis de la situación de salud de niños, niñas y adolescentes de escuelas de nivel primario de la República Argentina. [Consultado en diciembre 2019]. Accesible en: http://www.msal.gob.ar/images/stories/bes/graficos/0000000734cnt-reporte-2014-2017.pdf

29. Informe Dirección Nacional de Salud Bucal, DiNaSaB 2017. [Consultado en enero 2020]. Accesible en: https:// www.argentina.gob.ar/salud/bucodental

30. Organización Mundial de la Salud. Atención Primaria de Salud: Informe de la Conferencia Internacional sobre Atención Primaria de Salud, Alma-Ata, URSS, 6-12 de septiembre de 1978. Ginebra: OMS; 1978.

31. Organización Panamericana de la Salud, Organización Mundial de la Salud. Propuesta de Plan Regional Decenal sobre salud bucodental (CE138/14) [Consultado en enero 2020]. Accesible en: https://www.paho.org/spanish/gov/ce/ce138-fr-s.pdf 